\title{
The in vitro effect of nebulised hypertonic saline on human bronchial epithelium
}

\author{
Jennifer L. Goralski ${ }^{1,2,3}$, Dan Wu ${ }^{1}$, William R. Thelin ${ }^{4}$, Richard C. Boucher ${ }^{1,2}$ and \\ Brian Button ${ }^{1,5}$
}

Affiliations: ${ }^{1}$ Cystic Fibrosis Research and Treatment Center/Marsico Lung Institute, Chapel Hill, NC, USA. ${ }^{2}$ Division of Pulmonary and Critical Care Medicine, Dept of Medicine, University of North Carolina at Chapel Hill, Chapel Hill, NC, USA. ${ }^{3}$ Division of Pediatric Pulmonology, Dept of Pediatrics, University of North Carolina at Chapel Hill, Chapel Hill, NC, USA. ${ }^{4}$ Parion Sciences, Durham, NC, USA. ${ }^{5}$ Dept of Biochemistry and Biophysics, University of North Carolina at Chapel Hill, Chapel Hill, NC, USA.

Correspondence: Jennifer L. Goralski, Marsico Lung Institute, Campus Box 7248, 125 Mason Farm Rd, Chapel Hill, NC 27599, USA. E-mail: Jennifer_goralskiämed.unc.edu

@ERSpublications

This study provides insight into the magnitude of effect of hypertonic saline on airway surface hydration in muco-obstructed diseases http://ow.ly/FAVd30iR9Bj

Cite this article as: Goralski JL, Wu D, Thelin WR, et al. The in vitro effect of nebulised hypertonic saline on human bronchial epithelium. Eur Respir J 2018; 51: 1702652 [https://doi.org/10.1183/13993003.026522017].

ABSTRACT Inhaled hypertonic saline (HS) is an effective therapy for muco-obstructive lung diseases. However, the mechanism of action and principles pertinent to HS administration remain unclear.

An in vitro system aerosolised HS to epithelial cells at rates comparable to in vivo conditions. Airway surface liquid (ASL) volume and cell height responses were measured by confocal microscopy under normal and hyperconcentrated mucus states.

Aerosolised HS produced a rapid increase in ASL height and decrease in cell height. Added ASL volume was quickly reabsorbed following termination of nebulisation, although cell height did not recover within the same time frame. ASL volume responses to repeated HS administrations were blunted, but could be restored by a hypotonic saline bolus interposed between HS administrations. HS-induced ASL hydration was prolonged with hyperconcentrated mucus on the airway surface, with more modest reductions in cell volume.

Aerosolised HS produced osmotically induced increases in ASL height that were limited by active sodium absorption and cell volume-induced reductions in cell water permeability. Mucus on airway surfaces prolonged the effect of HS via mucus-dependent osmotic forces, suggesting that the duration of action of HS is increased in patients with hyperconcentrated mucus. 


\section{Introduction}

The effectiveness of mucociliary clearance is heavily dependent on adequate hydration of mucus. Under normal circumstances, airway epithelial cells have the capacity to absorb and secrete ions via active ion transport, with movement of water governed by transepithelial osmotic gradients. The net effect of this balance between secretion and absorption is to maintain mucus layer hydration at levels adequate to promote efficient mucus clearance [1]. Hyperconcentrated mucus, with failed mucus clearance, is characteristic of many muco-obstructive diseases, including cystic fibrosis (CF) [2], primary ciliary dyskinesia [3] and non-CF bronchiectasis [4].

Aerosolised hypertonic saline (HS) is an effective therapy in adults with $\mathrm{CF}[5,6]$ and non-CF bronchiectasis $[7,8]$, producing improvements in mucociliary clearance, forced expiratory volume in $1 \mathrm{~s}$ and quality of life. Despite its clinical utility, the mechanism of action of HS remains speculative. Accelerating mucociliary clearance via electrostatic interactions with mucins [9], expanding airway surface liquid (ASL) hydration [10] or inhibiting epithelial sodium channels (ENaC) [9] have been proposed as mechanisms. Most mechanistic studies deposited large volumes of HS onto human bronchial epithelial (HBE) surfaces and have not mimicked in vivo aerosol deliveries. Furthermore, most studies using cultured HBE cells were performed without endogenous normal ( $\%$ solids) or hyperconcentrated ( $\%$ solids) mucus on HBE surfaces $[5,10]$.

As prescribed for clinical use, HS is aerosolised to the lower airway surfaces in small $\left(\mathrm{nL} \cdot \mathrm{cm}^{-2} \cdot \mathrm{min}^{-1}\right)$ volumes over protracted intervals ( $\sim 15 \mathrm{~min})$. In this study, an in vitro aerosolisation system was developed to mimic in vivo aerosol delivery rates to airway epithelia that exhibited a range of mucus concentrations, spanning "normal" to muco-obstructive conditions. The primary goal was to characterise the kinetics of HS effects on airway surface hydration. The ancillary goal was to identify strategies to improve the efficacy of HS in the treatment of lung disease. Because of the great diversity of nebulisers and HS strengths used in clinical practice, and the fact that the osmotic effectiveness of HS depends on the rate of sodium chloride $(\mathrm{NaCl})$ delivery to airway surfaces, our studies are described in $\mu \mathrm{g} \mathrm{NaCl}$ deposited $\mathrm{cm}^{-2} \cdot \mathrm{min}^{-1}$, rather than percentage HS at a given deposition rate. Some of these data have been reported previously in the form of an abstract [11-15].

\section{Methods}

Full details of the methods are provided in the online supplementary material.

\section{HBE cultures and apical mucus content}

Primary HBE cells generated from cells isolated from explanted lungs were maintained at an air-liquid interface until fully differentiated. Culture surfaces were washed daily to produce HBE cultures with small amounts of residual mucus that mimicked normal epithelia, whereas HBE preparations with the mucus layer left unwashed for 2 weeks were generated to produce higher mucus compositions.

\section{Confocal measurements of ASL}

Primary HBE cells were labelled with $2.0 \mu \mathrm{M}$ calcein-AM (Invitrogen, Carlsbad, CA, USA) basolaterally. ASL was visualised by adding a $5 \mu \mathrm{L}$ of Texas red dextran $\left(70000 \mathrm{MW}, 5 \mathrm{mg} \cdot \mathrm{mL}^{-1}\right.$; Invitrogen) luminally. HS (7\%) solution was aerosolised utilising a vibrating mesh nebuliser (Aeroneb Lab; Aerogen, Galway, Republic of Ireland), modified to deliver small volumes (nL. $\mathrm{min}^{-1}$ ). Nebulisers were cleaned daily in accordance with Cystic Fibrosis Foundation guidelines and tested periodically for functionality. The system was mounted in an environmental chamber that controlled air currents and regulated temperature at $37^{\circ} \mathrm{C}$, humidity at $>50 \%$ and carbon dioxide at $5 \%$, interfaced to a scanning confocal microscope (SP5; Leica, Wetzlar, Germany). Cell and ASL heights were measured by high-speed XZ confocal scanning. Following baseline imaging, 7\% HS was nebulised to apical surfaces of HBE cells at the prescribed rate and effects were measured at $30-$ s intervals.

\section{ELISA determination of interleukin-8 secretion}

Production of interleukin (IL)-8 in HBE was measured $24 \mathrm{~h}$ after nebulising either 3,8 or $18 \mu \mathrm{g}$ $\mathrm{NaCl} \cdot \mathrm{cm}^{-2} \cdot \mathrm{min}^{-1}$ or sham nebulisation of PBS (control). For comparison, parallel cultures were treated with a bulk addition of hypertonic saline $(100 \mu \mathrm{L} 7 \%$ solution). Concentrations of IL- 8 in the culture medium were measured using a human IL-8 ELISA kit (R\&D Systems, Minneapolis, MN, USA) according to the manufacturer's protocol. Briefly, after incubation, the culture medium was removed. Samples and standards were added to wells of microplates precoated with an anti-human IL-8 monoclonal antibody and incubated for $2 \mathrm{~h}$. Each well was washed and incubated with the enzyme-linked polyclonal antibody specific for human IL-8 for $2 \mathrm{~h}$. The wells were washed to remove unbound antibody-enzyme reagent, substrate solution added to each well for $20 \mathrm{~min}$ at room temperature, the enzyme reaction was stopped, and IL- 8 concentrations determined by comparison of the optical density results with the standard curve. 


\section{Mathematical modelling}

The airway fluid transport model of WARREN et al. [16] was utilised in the construction of an integrative model of human airway ion and fluid transport $[17,18]$. This model solves an ordinary differential equation system for membrane potentials $(\mathrm{Va}, \mathrm{Vb})$, intracellular sodium, chloride and potassium ion concentrations $\left(\left[\mathrm{Na}^{+}\right] \mathrm{i},\left[\mathrm{Cl}^{-}\right] \mathrm{i},\left[\mathrm{K}^{+}\right] \mathrm{i}\right.$, respectively), extracellular ion concentrations $\left(\left[\mathrm{Na}^{+}\right] \mathrm{e},\left[\mathrm{Cl}^{-}\right] \mathrm{e},\left[\mathrm{K}^{+}\right] \mathrm{e}\right)$ and extracellular and intracellular fluid volumes (We, $\mathrm{W}_{\mathrm{i}}$ ) [18]. For the HS simulations, water with a high concentration of $\mathrm{NaCl}$ was "added" to the apical surface for designated volumes.

\section{Statistical analysis}

All data analyses and graphing were performed in SigmaPlot (Systat Software, San Jose, CA, USA). Mean values were compared via one-way ANOVA, with Holm-Sidak method for pairwise multiple comparisons in statistically significant findings. For non-normal data, the Kruskal-Wallis one-way ANOVA was used, with multiple groups compared via the Dunn method. Paired t-tests or Mann-Whitney rank sum tests were used where appropriate.

\section{Results}

The first series of studies focused on the effect of HS on HBE cultures with "normal" mucus concentrations ( $1.9 \pm 1.7 \%$ solids) on apical surfaces. HS was delivered to HBE cultures for 15 min at a rate
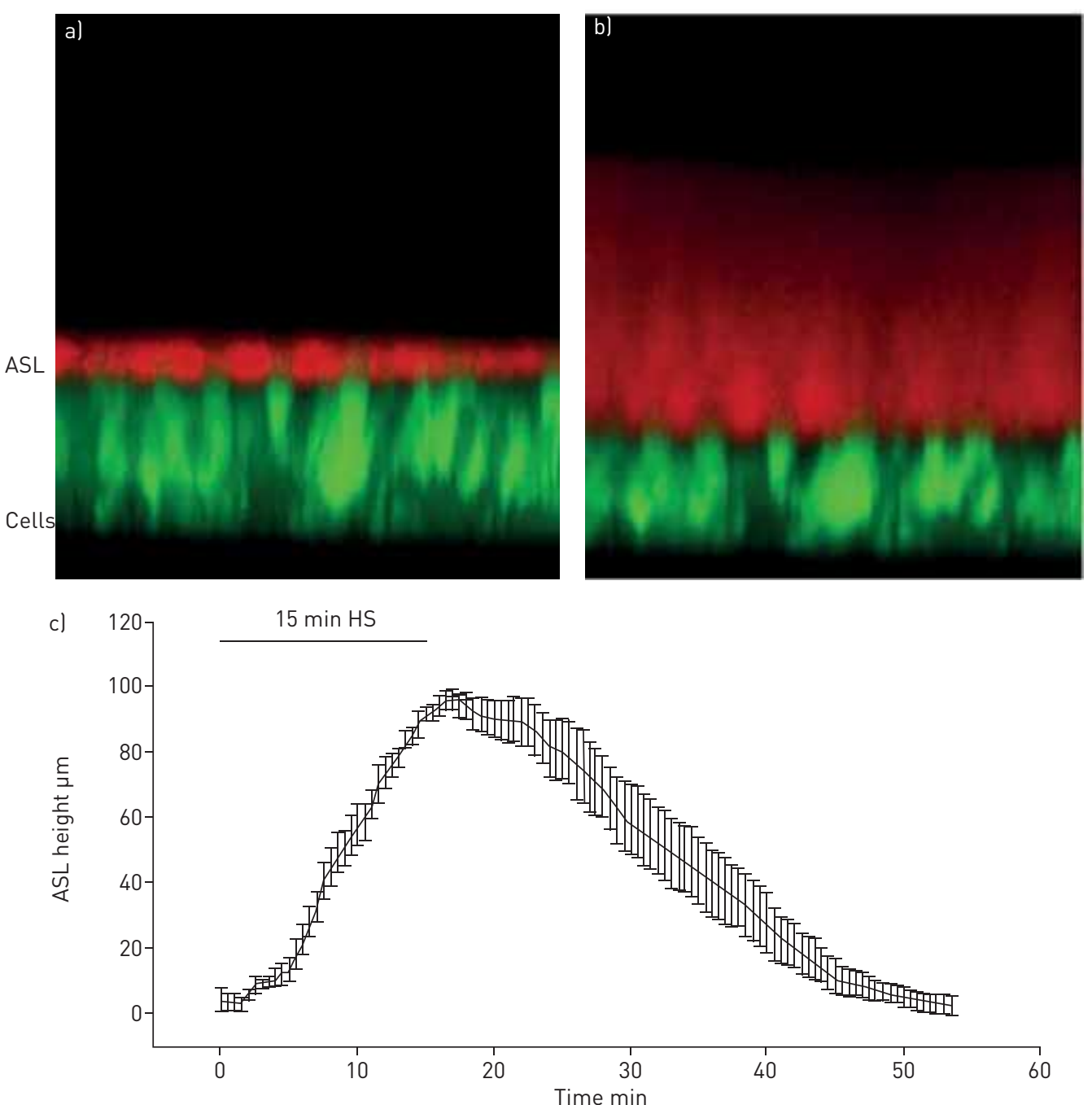

FIGURE 1 a) XZ-confocal image of a cross-section of airway epithelial cells stained with calcein, a fluorescent green dye, and airway surface liquid (ASL) stained with Texas red dextran, a cell-impermeable red dye; b) after administration of an osmotic stimulus (hypertonic saline (HS)), there is an increase in ASL height along with a decline in cell height; c) HS aerosolised to the surface of human bronchial epithelial cultures $\left(8 \mu \mathrm{g} \mathrm{NaCl} \cdot \mathrm{cm}^{-2} \cdot \mathrm{min}^{-1}\right.$ for $\left.15 \mathrm{~min}\right)$ results in a significant transient increase in ASL height, lasting $<1 \mathrm{~h} . \mathrm{n}=8$ for each experiment. 
of $8 \mu \mathrm{g} \mathrm{NaCl} \cdot \mathrm{cm}^{-2} \cdot \mathrm{min}^{-1}\left(7 \% \mathrm{HS}\right.$ delivered at $\left.110 \mathrm{~nL} \cdot \mathrm{cm}^{-2} \cdot \mathrm{min}^{-1}\right)$ to mimic a standard jet nebuliser delivery rate for human subjects (online supplementary material). A rapid and significant increase in ASL height was observed, reflecting the osmotic-driven transepithelial fluid flow in response to deposited HS [19] (figure 1). Net reabsorption of the osmotically expanded ASL began immediately after nebulisation was terminated, and ASL height returned to baseline height within $60 \mathrm{~min}$ after aerosol initiation.

In clinical practice, jet nebulisers typically deliver $\mathrm{HS}$ in a $4 \mathrm{~mL}$ dose over $\sim 15 \mathrm{~min}$, whereas vibrating mesh nebulisers deliver the same volume in $\sim 5 \mathrm{~min}$. To address whether HS delivery rates affect ASL volume responses, a constant total mass of salt was nebulised onto the HBE surface at varying nebulisation rates and, hence, durations of nebulisation. The rates selected for study were designed to a mimic delivery of $7 \%$ HS via jet nebuliser $\left(8 \mu \mathrm{g} \mathrm{NaCl} \cdot \mathrm{cm}^{-2} \cdot \mathrm{min}^{-1}\right)$, a vibrating mesh nebuliser $\left(18 \mu \mathrm{g} \mathrm{NaCl} \cdot \mathrm{cm}^{-2} \cdot \mathrm{min}^{-1}\right)$ and an arbitrarily defined "slow" nebuliser $\left(3 \mu \mathrm{g} \cdot \mathrm{cm}^{-2} \cdot \mathrm{min}^{-1}\right.$ ) (figure 2a). Both the 8 and $18 \mu \mathrm{g}$ $\mathrm{NaCl} \cdot \mathrm{cm}^{-2} \cdot \mathrm{min}^{-1}$ rates produced rapid and significant increases in ASL height. The response of cultures to the $3 \mu \mathrm{g} \mathrm{NaCl} \cdot \mathrm{cm}^{-2} \cdot \mathrm{min}^{-1}$ rate was considerably smaller with respect to ASL volume expansion, but longer due to the extended nebulisation duration. All cultures, irrespective of nebulisation rate, rapidly absorbed the added volume from the ASL once nebulisation was terminated.

Areas under the curves (AUCs) of the ASL height data were calculated as an index of "hydration activity" of the nebulised solution. Interestingly, faster salt deposition $\left(18 \mu \mathrm{g} \cdot \mathrm{cm}^{-2} \cdot \mathrm{min}^{-1}\right)$ produced a lower, though not statistically different, total ASL hydration compared to standard jet nebuliser rates $\left(8 \mu \mathrm{g} \cdot \mathrm{cm}^{-2} \cdot \mathrm{min}^{-1}\right)$. Slower aerosol deposition $\left(3 \mu \mathrm{g} \cdot \mathrm{cm}^{-2} \cdot \mathrm{min}^{-1}\right)$ produced trends towards lower peak and AUC values compared to the faster rates (figure $2 b$ ).
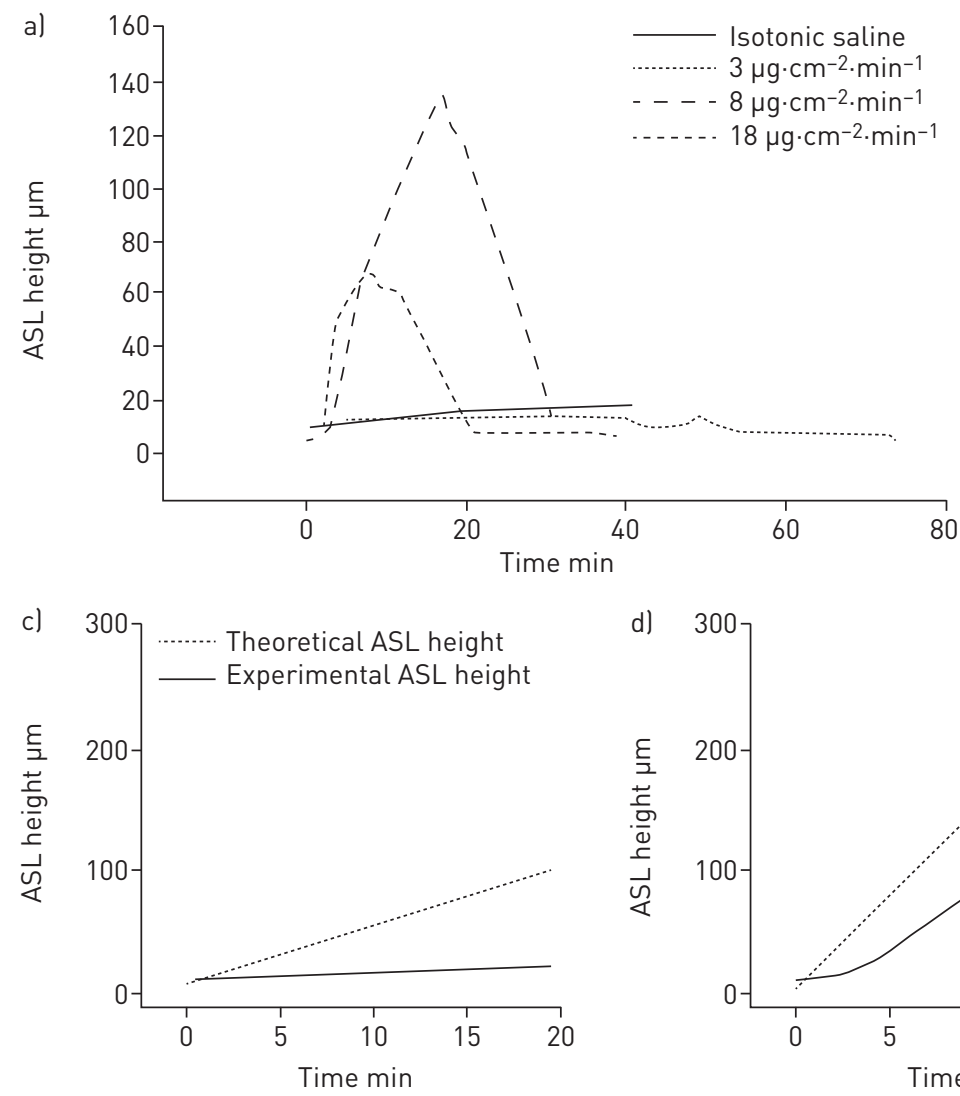

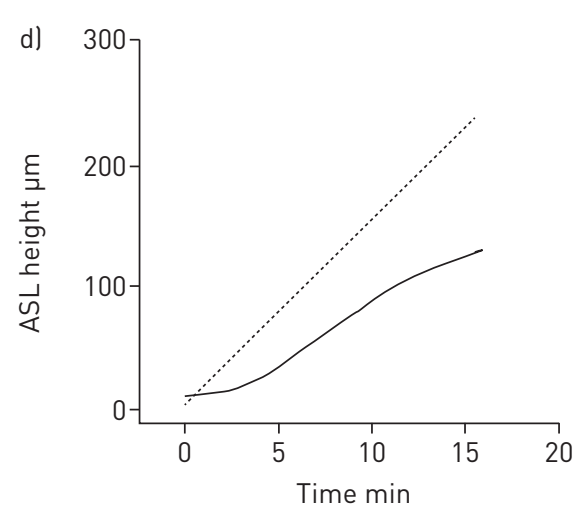

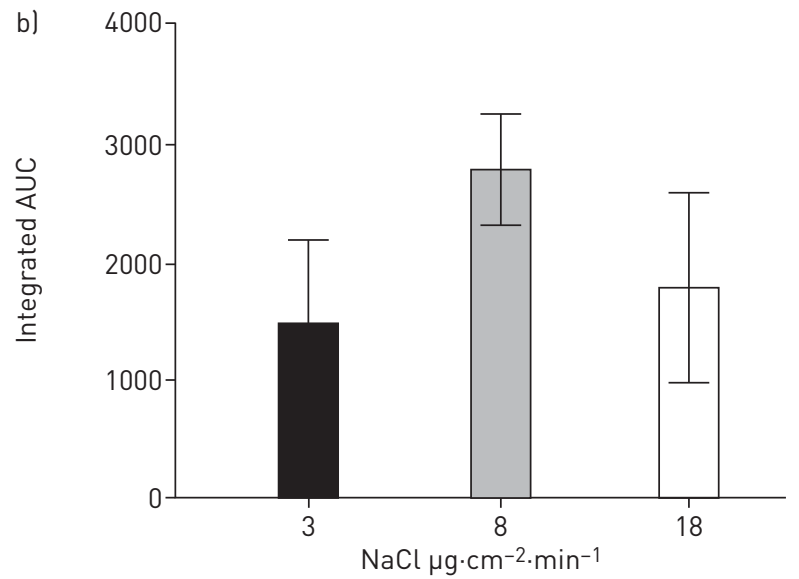

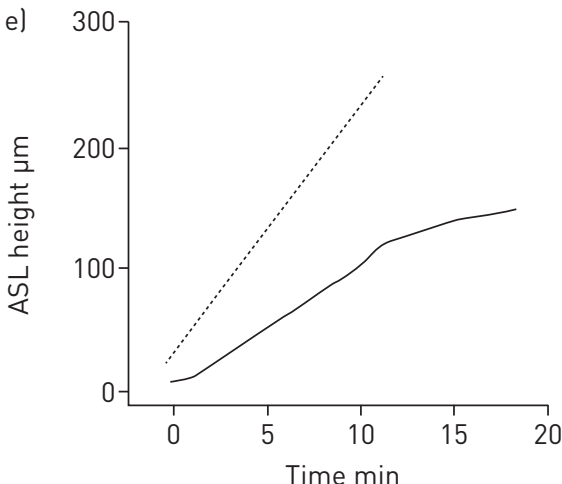

FIGURE 2 Effects of variation in hypertonic saline (HS) delivery rates on airway surface liquid (ASL) volume responses. a) Comparison of ASL heights achieved with three rates of nebulised HS (low $3 \mu \mathrm{g} \mathrm{NaCl} \cdot \mathrm{cm}^{-2} \cdot \mathrm{min}^{-1} ;$ moderate $8 \mu \mathrm{g} \mathrm{NaCl} \cdot \mathrm{cm}^{-2} \cdot \mathrm{min}^{-1}$; and high $18 \mu \mathrm{g} \mathrm{NaCl} \cdot \mathrm{cm}^{-2} \cdot \mathrm{min}^{-1}$ ) with duration varied to hold constant total delivered mass of salt. Isotonic saline shown as control. $p<0.05$ for all groups compared to isotonic saline control. b) Both absolute height change and total hydration (integrated area over time) favoured the mid-range dosing regimen, but did not reach statistical significance $(p=0.373) . c, d, e)$ Theoretical heights indicate the expected rise in ASL height if all salt deposited resulted in an equimolar flux of water into the ASL. Experimental ASL height lines indicate the actual change in ASL height observed during experiments. The failure of these two lines to overlay indicates an inhibition of the transport of water from the epithelial cells into the ASL. c) Low-dose nebulisation $\left(3 \mu \mathrm{g} \mathrm{NaCl} \cdot \mathrm{cm}^{-2} \cdot \mathrm{min}^{-1}\right)$ : theoretical slope $4.72 \mu \mathrm{m} \cdot \mathrm{min}^{-1}$ versus experimental slope of $0.45 \mu \mathrm{m} \cdot \mathrm{min}^{-1}(\mathrm{p}<0.001)$; $\left.d\right)$ medium-dose nebulisation $(8$ $\mu \mathrm{g} \mathrm{NaCl} \cdot \mathrm{cm}^{-2} \cdot \mathrm{min}^{-1}$ ): theoretical slope $14.92 \mu \mathrm{m} \cdot \mathrm{min}^{-1}$ versus experimental slope of $8.66 \mu \mathrm{m} \cdot \mathrm{min}^{-1}$ ( $\left.p<0.001\right)$; e) high-dose nebulisation (18 $\mu \mathrm{g}$ $\mathrm{NaCl} \cdot \mathrm{cm}^{-2} \cdot \mathrm{min}^{-1}$ ): theoretical slope $51.49 \mu \mathrm{m} \cdot \mathrm{min}^{-1}$ versus experimental slope of $20.78 \mu \mathrm{m} \cdot \mathrm{min}^{-1}(\mathrm{p}<0.001)$. $\mathrm{n}=4$ per experimental condition. 
Assuming no active $\mathrm{Na}^{+}$-mediated volume absorption or change in apical membrane water permeability during aerosolisation, deposition of a constantly accumulating mass of salt to the surface of airway epithelia is predicted to produce a linear increase in ASL volume. For all rates studied, the measured ASL height deviated consistently from the predicted height (figure $2 \mathrm{c}-\mathrm{e}$ ). The deviation of measured ASL height from predicted values could reflect active $\mathrm{Na}^{+}$absorption, reductions in HBE water permeabilities that govern rates of osmotically driven water flow to the HBE surface, or both.

With respect to the role of $\mathrm{Na}^{+}$transport on ASL volume responses to $\mathrm{HS}$ administration, we speculated that $\mathrm{Na}^{+}$transport would modify the magnitude of HS-induced ASL volume expansion immediately after initiation of HS administration. To experimentally investigate this possibility, ASL volume responses to the low rate of $\mathrm{HS}$ administration $\left(3 \mu \mathrm{g} \mathrm{NaCl} \cdot \mathrm{cm}^{-2} \cdot \mathrm{min}^{-1}\right)$ were measured in the presence and absence of a selective ENaC blocker (VX-371; Vertex Pharmaceuticals, Boston, MA, USA). As compared to HS alone, the co-administration of VX-371 $\left(50 \mu \mathrm{g} \cdot \mathrm{mL}^{-1}\right)$ produced a more rapid and sustained ASL response during nebulisation (figure 3a). A mathematical model of airway epithelial ion and water transport quantitatively analysed the relationship between HS-mediated changes to ASL height and active $\mathrm{Na}^{+}$absorption in the presence and absence of $\mathrm{ENaC}$ blockade (figure $3 \mathrm{~b}$ ). The fit of the model to experimental data describing ASL response for $\mathrm{HS}$ administration in the absence of $\mathrm{ENaC}$ inhibition was achieved by maintaining $\mathrm{Na}^{+}$ transport at basal rates during $\mathrm{HS}$ aerosolisation and increasing $\mathrm{Na}^{+}$transport roughly five-fold during the re-absorptive phase. The mathematical model replicated the effect of ASL kinetics with ENaC inhibition, with a more rapid rate of accumulation and overshoot of ASL height, consistent with inhibition of active $\mathrm{Na}^{+}$absorption during HS administration.

Previous studies have demonstrated that HS can produce a reduction in cell volume [20, 21], which can then inhibit cell water permeabilities [21-23]. Additionally, studies suggest that administration of hypertonic saline also will result in inflammatory changes within the airway epithelia, particularly IL-8, a known neutrophil chemoattractant [24-26]. Accordingly, we measured HBE cell volume responses and IL-8 production to HS aerosol administration to test whether reductions in cell water permeabilities blunted ASL responses to aerosolised HS and/or induced pro-inflammatory cytokines. Figure 4a shows that cell height (a surrogate for cell volume) was reduced during and after HS nebulisation by 20\% from initial cell heights $(\mathrm{p}<0.01)$. In contrast, cell volume was not affected by nebulisation of isotonic saline. These data suggest that deposition of luminal HS osmotically draws water from cells onto the surface via apical membrane water channels. Figure $4 \mathrm{~b}$ demonstrates cumulative IL- 8 production by HBE $24 \mathrm{~h}$ after nebulising HS or PBS control to airway surfaces. A stepwise increase in IL-8 production was noted with increasing doses of HS compared with PBS control (statistically different from control at $18 \mu \mathrm{g}$ dose and bulk dosing).

The contribution of cellular water permeability to ASL volume responses was investigated further using a pharmacological approach. Because mercury-sensitive water channels (aquaporins) have been identified in the apical and basolateral membranes of $\mathrm{HBE}$ epithelia $[27,28]$, the role of these channels on HS-mediated changes in ASL volume responses was evaluated experimentally. Because mercury has off-target toxic effects with long exposure, the protocols involved acute exposures to mercury and HS [29].
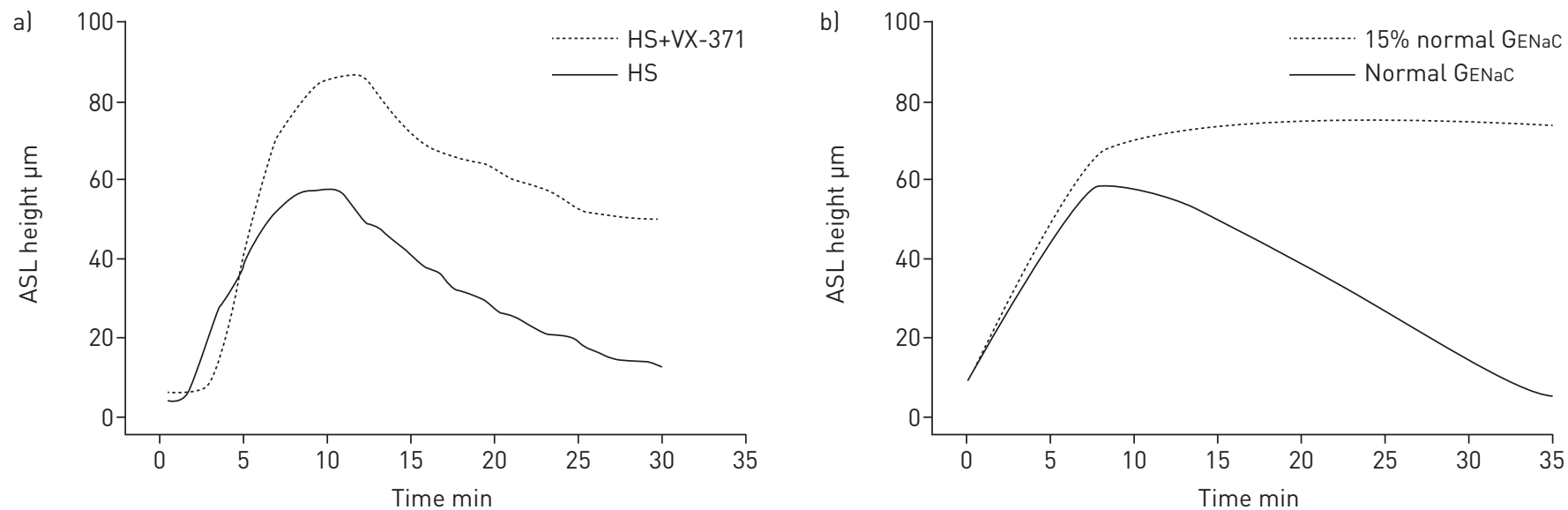

FIGURE 3 Effect of sodium reabsorption on hypertonic saline (HS)-mediated airway surface liquid (ASL) height. a) Experimental data of hypertonic saline (at $8 \mu \mathrm{g} \mathrm{NaCl} \cdot \mathrm{cm}^{-2} \cdot \mathrm{min}^{-1}$ ) in the absence and presence of a potent sodium channel blocker (VX-371 at $50 \mu \mathrm{g} \cdot \mathrm{mL}^{-1}$ ); b) $\mathrm{mathematical} \mathrm{model}$

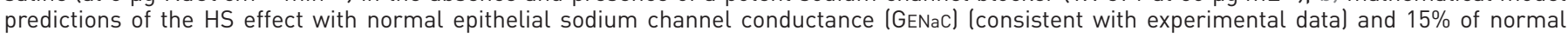
GENaC. 

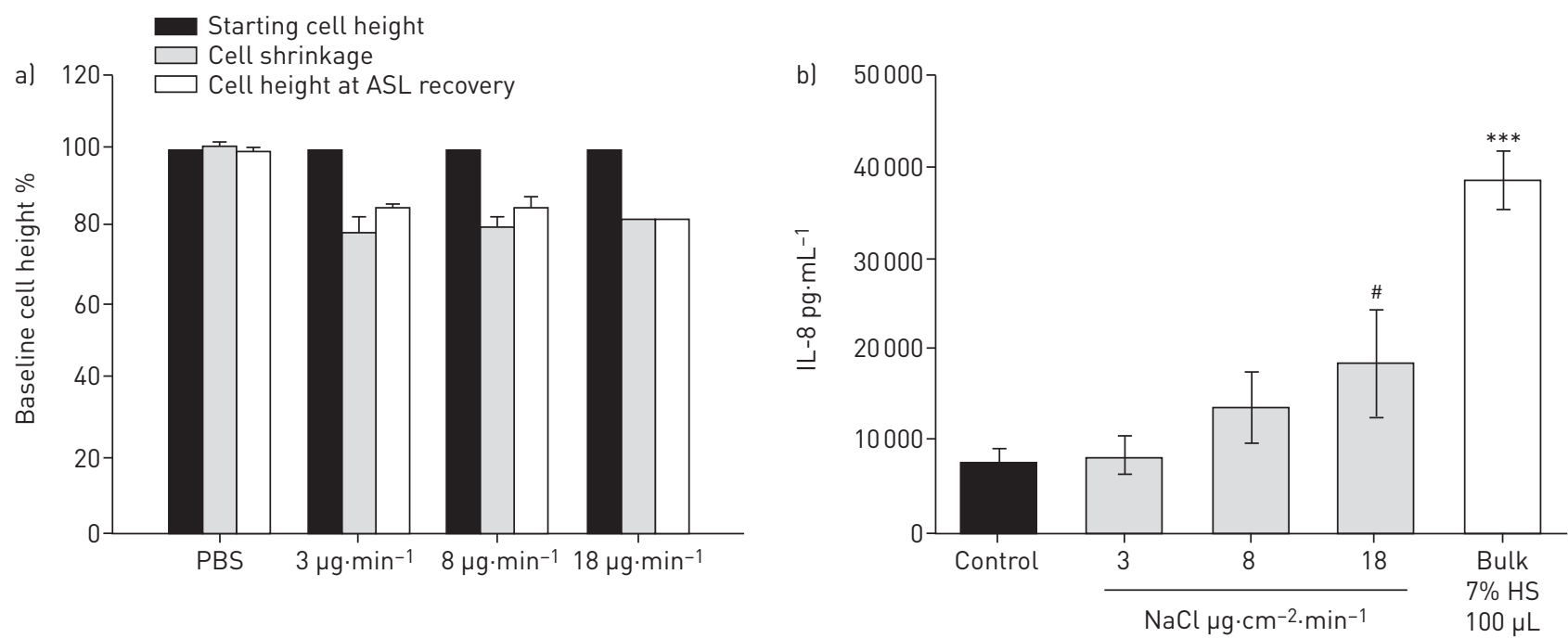

FIGURE 4 a) Cell volume changes in response to osmotic stimulus of hypertonic saline (HS) at varying HS deposition rates. A $20 \%$ decrease in cell height in response to the hypertonic stimulus was observed at all rates, which was significantly different from baseline and isotonic (PBS) saline administration ( $p<0.01$ by Holm-Sidak method). Cell height did not recover during the time frame of the experiment (60 min). Cell volume reduction was a consistent finding regardless of rate of HS administration. $n=4$ for each condition. b) Production of interleukin (IL)-8 in response to increasing doses of nebulised or bulk HS. Statistically different from control (PBS) at the $18-\mu \mathrm{g}$ dose and bulk dosing (100 $\mu \mathrm{L}$ of $7 \%$ HS). \#: $p<0.002$ by Holm-Sidak method; ${ }^{* * *}$ : $p<0.001$.

As shown in figure 5a, both apical and basolateral mercury chloride $\left(\mathrm{HgCl}_{2}\right)$ administration significantly reduced ASL volume responses to aerosolised HS. Inhibition of apical aquaporins resulted in a smaller decrease in cell height compared to basolateral aquaporin inhibition. These data suggest that in the setting of aerosolised HS, cell volume homeostasis is dominated by the apical membrane water permeability (figure 5b). Modelling studies mimicked the experimental data (figure 5c).

We hypothesised that persistent reduction in cell volumes observed after HS administration would limit the ability of HBEs to respond to repeated administrations of HS. This hypothesis was tested by exposing HBE cultures to sequential HS administrations $\left(8 \mu \mathrm{g} \mathrm{NaCl} \cdot \mathrm{cm}^{-2} \cdot \mathrm{min}^{-1}\right)$, with the second administration delivered 15 min after ASL height had returned to baseline following the first HS dose. ASL height and AUC of the second administration was significantly reduced compared to initial HS administration (figure 6a).

Next, we directly tested the hypothesis that the reduction in the effectiveness of the second HS administration reflected reduced HBE cell water permeabilities consequent to cell volume reduction, rather than persistent acceleration of $\mathrm{Na}^{+}$transport. To test this notion, a small volume of hypotonic saline $(0.63 \% \mathrm{NaCl}, 10 \mu \mathrm{L})$ was transiently added $(3 \mathrm{~min})$ to the apical surface of HBEs following the first aerosolised HS administration. Application of the hypotonic saline bolus resulted in cell swelling (increase in height $32 \pm 9 \mu \mathrm{m}$ ) that restored cell height to baseline levels. The interposition of the hypotonic bolus was associated with a statistically larger response in ASL and AUC to the second HS administration (figure $6 \mathrm{~b})$. In contrast, applying a bolus dose of isotonic saline $(0.9 \% \mathrm{NaCl}, 10 \mu \mathrm{L})$ produced neither cell swelling nor a recovery in responsiveness to the subsequent HS challenge (figure 6c). Modelling this phenomenon predicted that reduction in apical water permeability to $\sim 10 \%$ of baseline levels would produce the AUC decrement with the second HS administration observed experimentally (figure 6d).

Next, we investigated whether the presence of hyperconcentrated mucus associated with muco-obstructive diseases [30] alters the kinetics of HS-induced hydration, utilising HBE cultures exhibiting hyperconcentrated mucus $(12 \pm 4.3 \%$ solids). CF is the disorder best characterised by hyperconcentrated mucus. However, other muco-obstructive diseases, e.g. primary ciliary dyskinesia [3] and non-CF bronchiectasis [4] exhibit hyperconcentrated mucus, and we have previously shown that CF cells and normal HBE in the presence of hyperconcentrated mucus behave similarly in response to nebulised hypertonic saline [13]. Accordingly, we focused our studies of muco-obstructive disease mucus on normal HBE with hyperconcentrated mucus to make the data relevant to other possible muco-obstructive lung diseases. Pre-HS administration, ASL height in the hyperconcentrated mucus cultures was approximately three-fold higher than the normal mucus cultures. Notably, the changes in ASL height with HS administration were substantially increased in hyperconcentrated mucus cultures as compared to normal mucus cultures (figures 1 and 7a). Although both normal and hyperconcentrated mucus cultures absorbed 

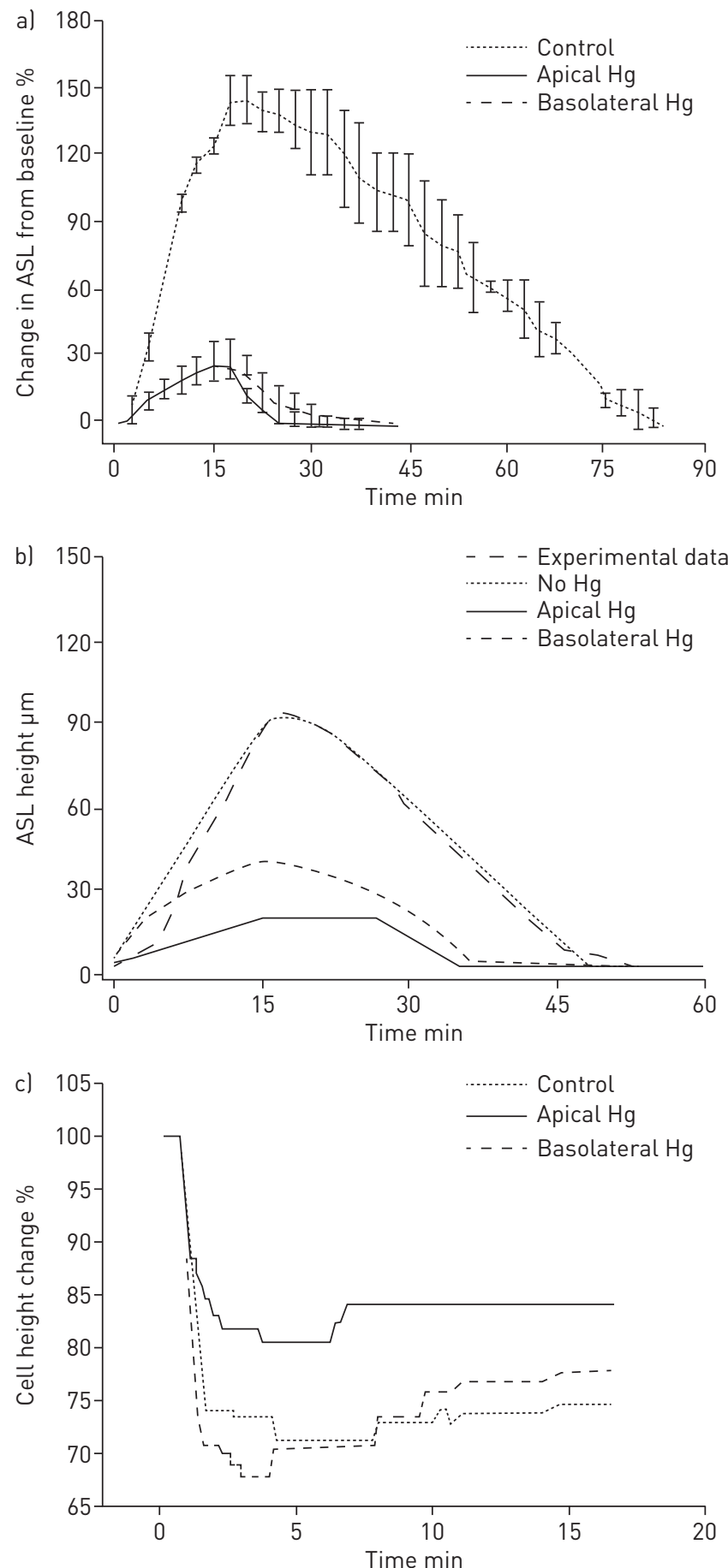

FIGURE 5 Incubation with mercury $(\mathrm{Hg})$ chloride apically or basolaterally resulted in a diminished airway surface liquid (ASL) response to aerosolised hypertonic saline (HS) (at $8 \mu \mathrm{g} \mathrm{NaCl} \cdot \mathrm{cm}^{-2} \cdot \mathrm{min}^{-1}$ ) compared with native cultures. a) Experimental data. $\mathrm{p}<0.001$ via Holm-Sidak method for comparisons to control. b) Mathematical model of experimental data. $n=8$ for each condition. c) Cell height change to aerosolised HS in the absence (control) or presence of selective block of the apical or basolateral cell membrane with mercury chloride ( $p<0.001$ for all comparisons).

the increased ASL volume immediately after cessation of nebulisation, the rate of reabsorption was slower in hyperconcentrated versus normal mucus cultures (5.5 versus $2.8 \mu \mathrm{m} \cdot \mathrm{min}^{-1}, \mathrm{p}=0.052$; figure $7 \mathrm{~b}$ ). In the hyperconcentrated mucus cultures, the total duration of HS-induced ASL volume expansion was approximately double that of normal mucus cultures. Further, in repetitive HS administration protocols, the second administration of HS produced an ASL response similar to the first administration (figure 7c). Pertinent to this observation, cell heights in HBEs covered by hyperconcentrated mucus decreased less in 

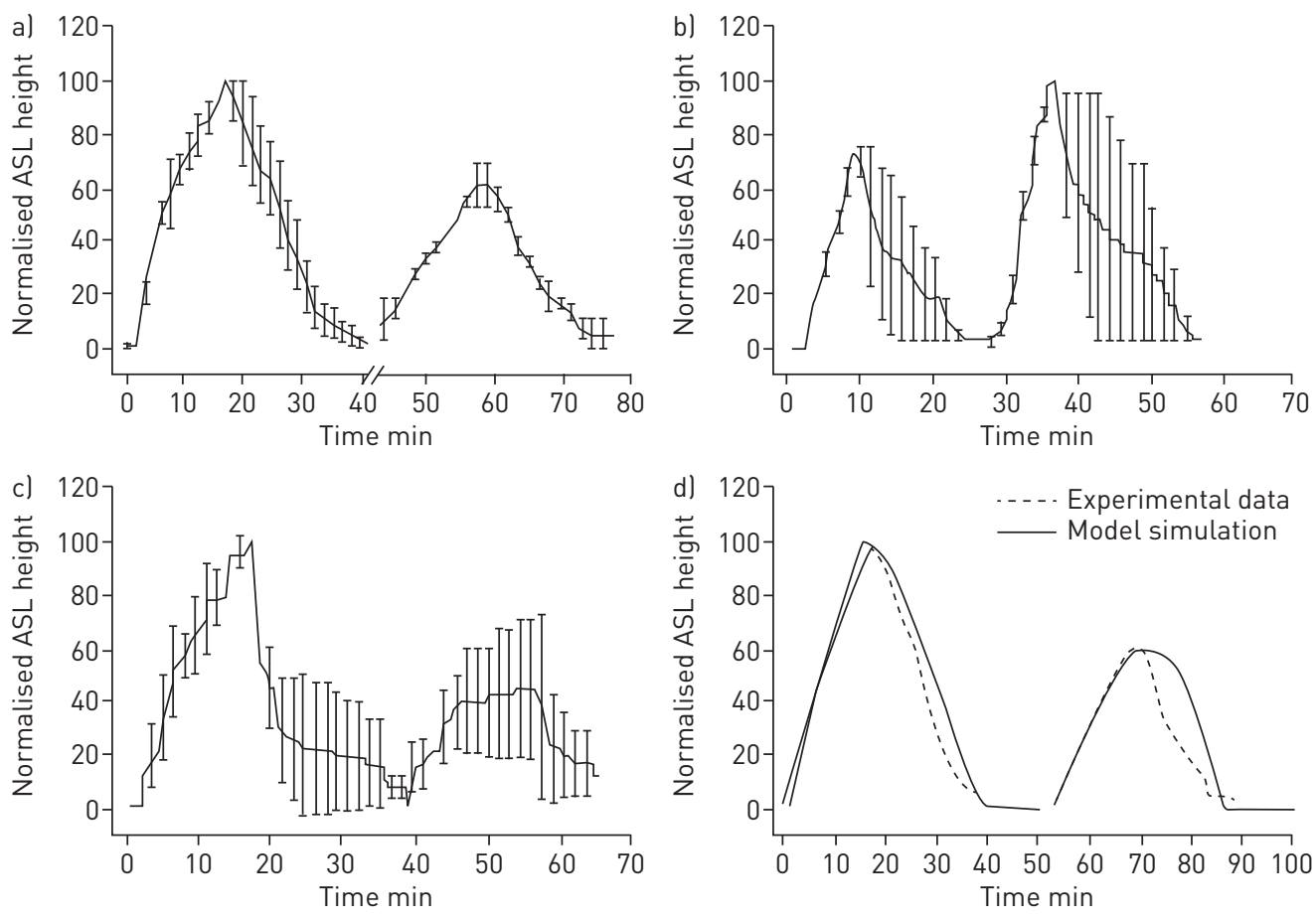

FIGURE 6 Sequential airway surface liquid (ASL) volume responses to aerosolised $7 \%$ hypertonic saline (HS) for $15 \mathrm{~min}\left(8 \mu \mathrm{g} \mathrm{NaCl} \cdot \mathrm{cm}^{-2} \cdot \mathrm{min}^{-1}\right)$. a) Sequential ASL volume responses to aerosolised HS. A smaller ASL response to a second administration of aerosolised HS was observed when two identical HS doses were separated by $15 \mathrm{~min}$. Break in x-axis indicates time between two doses. For each dose, HS was nebulised at $8 \mu \mathrm{g} \mathrm{NaCl} \cdot \mathrm{cm}^{-2} \cdot \mathrm{min}^{-1}$ and administered continuously for $15 \mathrm{~min}$. The ASL height of the second peak was $\sim 60 \%$ of the first peak. $p<0.001$ by Mann-Whitney rank sum test. b) A hypotonic saline rinse was interposed between HS administration. The hypotonic solution administration produced cellular swelling associated with improved ASL volume responses to a subsequent administration of HS ( 140\% of first dose). The difference in response was statistically significant ( $p=0.002$ via Mann-Whitney rank sum test) in favour of the post-hypotonic peak. c) Interposition of an isotonic rinse between the first and second HS administration. The same second-dose effect is not seen when doses of HS were separated by an isotonic saline bolus ( $p=0.78$ by Mann-Whitney rank sum test). ASL height of second peak persisted at $\sim 50 \%$ of the first peak. d) In a model simulation, the water permeability of the human bronchial epithelial apical membrane during the second dose was reduced to $10 \%$ of the basal water permeability. $n=4$ for each condition.

response to HS $(15 \pm 4.7 \%$ versus $23 \pm 5.7 \%$ ) than in normal cultures, although this difference did not achieve statistical significance.

The larger peak ASL response and slower absorption of $\mathrm{NaCl}$ and water in the hyperconcentrated versus normal mucus cultures suggested that an additional force was governing water flux in the hyperconcentrated mucus system. To generate an index of the magnitude of this effect, the predicted versus measured ASL heights during HS administration were compared (figure $7 \mathrm{~d}$ ). The measured early administration values were significantly closer to the predicted value for hyperconcentrated compared with normal mucus cultures (figure $2 \mathrm{c}-\mathrm{e}$ ), suggesting that the presence of concentrated mucus generated additional mucus-related osmotic forces that retarded fluid absorption [30].

\section{Discussion}

Our in vitro studies of clinically relevant rates of aerosolised HS delivered to HBE cultures with $2 \%$ mucus solids revealed a rapid HS-induced expansion of ASL volume. This ASL expansion in response to HS aerosols is similar to that reported in vivo in mice measured by synchrotron-based tomography and in HBE cultures by optical coherence tomography [31, 32]. Furthermore, acute aerosolisation of HS has been reported to reduce airway mucus concentrations in chronic obstructive pulmonary disease subjects [33]. Thus, the ASL expansion findings, juxtaposed to our findings that the osmolarity of ASL during HS nebulisation probably does not exceed $370 \mathrm{mOsm} \cdot \mathrm{L}^{-1}$ (see later), argues that the major effect of $\mathrm{HS}$ on mucus clearance is via ASL volume expansion and mucus dilution.

The HS-induced ASL volume response was mediated by water flux in response to the deposition of osmotically active $\mathrm{NaCI}$ on $\mathrm{HBE}$ surfaces. Two observations suggest that aquaporin-mediated transepithelial water fluxes dominated the ASL response to aerosolised HS. First, the flux of water to the 

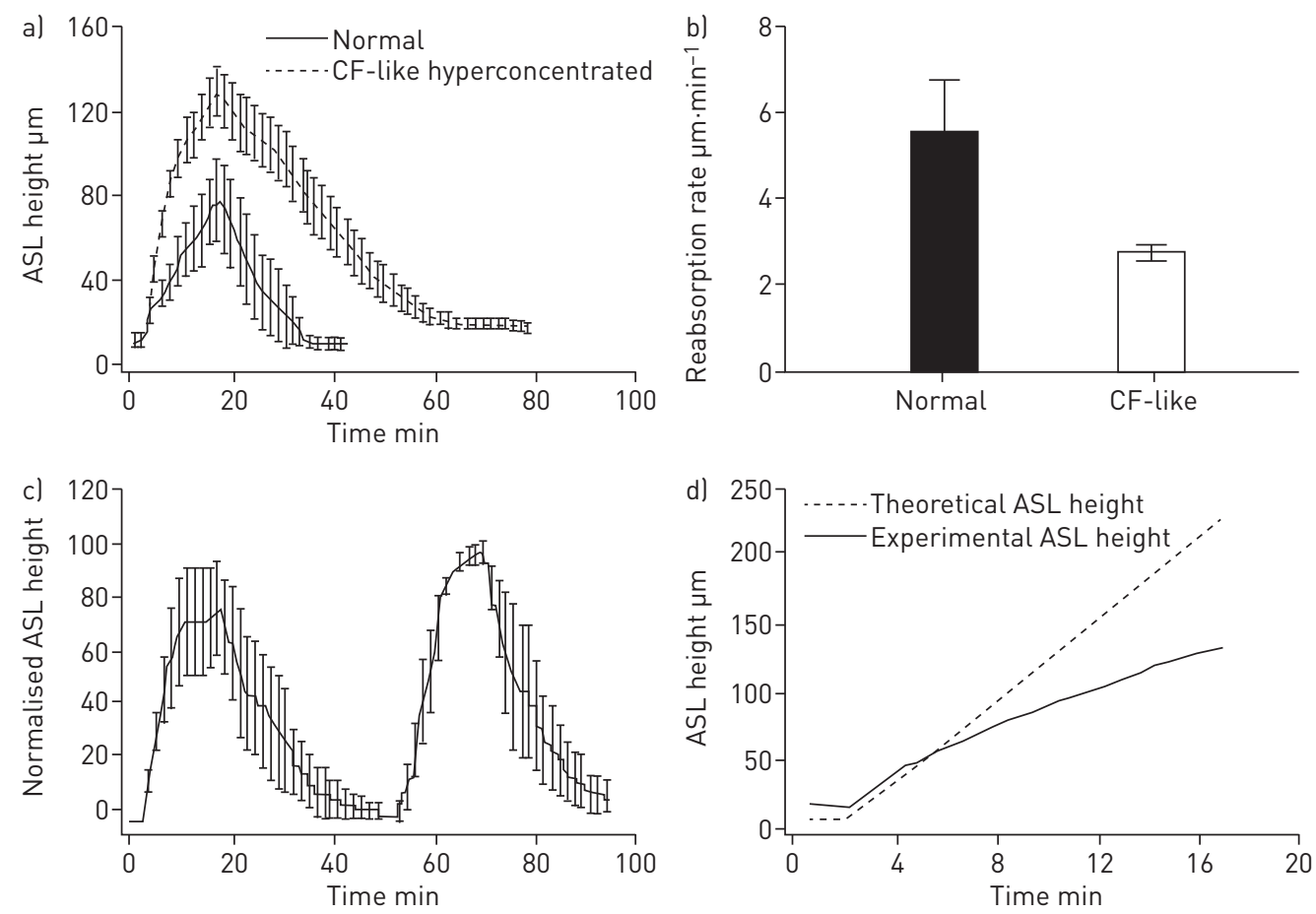

FIGURE 7 The effects of hyperconcentrated mucus on human bronchial epithelial (HBE) surfaces on nebulised hypertonic saline (HS) (at $8 \mu \mathrm{g} \mathrm{NaCl} \cdot \mathrm{cm}^{-2} \cdot \mathrm{min}^{-1}$ ) in inducing airway surface liquid (ASL) volume responses. a) In the normal ( $2 \%$ mucus) state, there was a three-fold increase in ASL volume during nebulisation. HBE cultures with hyperconcentrated mucus (12\%) (cystic fibrosis (CF)-like) exhibited a seven-fold increase in ASL above baseline ASL. In addition, the duration of ASL being increased above basal levels was increased in hyperconcentrated mucus HBE cultures. b) Rates of reabsorption (time to baseline ASL, in $\mu \mathrm{m} \cdot \mathrm{min}^{-1}$ ) were significantly different between the normal and the hyperconcentrated mucus cultures. $p=0.052$ by MannWhitney rank sum testing. c) Two sequential doses of HS administered to hyperconcentrated cultures. ASL height difference favours the second dose $(p<0.001)$. d) Theoretical expected rise in ASL height if all salt deposited remained on the surface and resulted in an equimolar flux of water into the ASL and the actual change in ASL height observed during experiments. Compare with figure $2 \mathrm{c}-\mathrm{e}$; in the presence of an intact mucus layer (12\% solids), actual and expected ASL height more closely approximated each other early in the HS delivery interval.

lumen in response to HS was accompanied by a reduction in cell height/volume, suggesting intracellular water moved into ASL. This notion is consistent with previous studies [21] that reported the aquaporin-dominated apical membrane water permeability of $\mathrm{HBE}$ was $\sim 10$-fold higher than the basolateral membrane. Note that this configuration permits the use of changes in cell volume as an "osmometer" to detect ASL osmolarity. The cell volume responses to HS aerosols suggest that ASL achieved osmolarities of $\sim 370 \mathrm{mOsm} \cdot \mathrm{L}^{-1}$ during $\mathrm{HS}$ administration (7\% $\mathrm{HS}$ given at $7.7 \mu \mathrm{g}$ $\mathrm{NaCl} \cdot \mathrm{cm}^{-2} \cdot \mathrm{min}^{-1}$ ), far less than achieved by direct HS additions. The second observation was that the ASL responses to aerosolised HS were blocked by $\mathrm{HgCl}_{2}$, an inhibitor of aquaporin 3-5 known to be expressed in HBEs. These data, along with the cell modelling data presented, strongly suggest that aquaporin-mediated cellular water permeabilities participate in the ASL volume responses to aerosolised HS (figure 4).

The effects of aerosolised HS on cell volume reduction and transepithelial $\mathrm{Na}^{+}$transport probably explain the discrepancy between the predicted ASL responses to deposition of HS on HBE surfaces and measured responses. First, reductions in cell volume were associated with reductions in cell water permeabilities, limiting water fluxes toward a hypertonic lumen. Second, as evidenced by a greater maximal response to $\mathrm{HS}$ administration in the presence of an $\mathrm{ENaC}$ blocker, active $\mathrm{Na}^{+}$transport removed a component of the deposited $\mathrm{NaCl}$ during aerosol administration. An interesting observation that emerged from the modelling of active $\mathrm{Na}^{+}$transport responses to $\mathrm{HS}$ was that an increase in $\mathrm{Na}^{+}$transport rates was required to mimic the rate of ASL volume absorption post-cessation of aerosol. This effect probably reflects dilution of local extracellular inhibitors of $\mathrm{ENaC}$, e.g. ATP, that accelerated the rate of absorption during HS administration [34].

A key issue with respect to HS therapeutic responses relates to the concentration of mucus on airway surfaces. The presence of hyperconcentrated mucus on HBE surfaces was associated with increased ASL 
heights, a longer duration of ASL hydration to a single HS administration, and larger responses to repetitive doses of HS. We postulate that mucus acts as a "sponge", providing a concentration-dependent polymer gel-mediated osmotic driving force, added to that of HS-induced osmotic gradients, which modulates maximal mucus/ASL heights on the HBE surface. The increased durability of the ASL expansion in response to aerosolised HS in hyperconcentrated mucus cultures parallels the longer duration of action of HS in CF as compared to normal subjects observed in in vivo mucociliary clearance studies $[5,35]$.

In addition, our studies were designed to identify strategies to increase the effectiveness of aerosolised HS to expand ASL hydration, including studies of HS delivery rates and repetitive dosing. We found that very slow rates of HS delivery were relatively ineffective. We speculate that this finding reflects the fact that $\mathrm{NaCl}$ deposition rates were similar to rates of endogenous active transepithelial $\mathrm{Na}^{+}$absorption [36]. Delivery of HS at rates approximating a jet nebuliser were effective in increasing ASL hydration, but a further increase in efficacy was not observed with faster rates mimicking vibrating mesh nebulisers. The absolute decrease in AUC and the slowing of ASL volume expansion observed towards the end of rapid HS administration $\left(18 \mu \mathrm{g} \mathrm{NaCl} \cdot \mathrm{cm}^{-2} \cdot \mathrm{min}^{-1}\right)$ are consistent with large reductions in transepithelial water permeabilities. Based on our IL-8 measurements and the pro-inflammatory nature of this cytokine, it is possible that faster rates of nebulisation could be detrimental.

An important observation pertinent to HS dosing frequency was that HBE cell heights did not return to baseline levels for $4 \mathrm{~h}$ following HS administration. We speculate that the absence of cell volume regulation mechanisms reflected decreased water permeabilities. It is likely that the delay in return of apical water permeability after HS accounted for the blunted response to second HS administrations on HBE cultures with normal mucus (figure 5). Importantly, epithelial cell swelling induced by hypotonic saline restored second HS responses. These data suggest that novel strategies may be required to optimise the effectiveness of repetitive HS dosing in patients with milder lung disease. In contrast, repetitive HS dosing in subjects with severe (12\% mucus solids) disease may be an effective strategy.

In conclusion, ASL volume of $\mathrm{HBE}$ cultures with normal mucus concentrations increased in response to nebulised HS delivered at rates similar to those delivered clinically, but reabsorption began immediately with termination of nebulisation. Neither nebulising faster nor slower improved ASL hydration. However, the effects of aerosolised HS were more pronounced and prolonged in HBE cultures with hyperconcentrated mucus, probably reflecting the increased osmotic forces generated by concentrated mucus. These data predict a prolonged duration of HS action in patients with muco-obstructive diseases.

Acknowledgements: The authors would like to acknowledge the University of North Carolina (UNC) Tissue Culture Core (NIH P30 DK065988 and CFF RDP BOUCHE15R0), as well as Eric Roe (UNC CF Research and Treatment Center, Chapel Hill, NC, USA) for his editorial assistance. This project was funded by the Cystic Fibrosis Foundation (CFF) Leroy Matthews Physician Scientist Award (CFF GORALS12LO) as well as BUTTON07XX0, NIH R01HL125280-01A1, and NIH P30DK065988-11.

Author contributions: Concept and design: J.L. Goralski and B. Button. Data acquisition: J.L. Goralski, B. Button and W.R. Thelin. Data modelling: D. Wu. Data interpretation, drafting and critical revision of the work: J.L. Goralski, R.C. Boucher and B. Button.

Conflict of interest: R.C. Boucher reports personal fees from Parion Sciences, outside the submitted work. W.R. Thelin is an employee of Parion Sciences and reports non-financial support, outside the submitted work; in addition, he has a patent - VX-371 is a patented molecule licensed to Vertex Pharmaceuticals.

Support statement: This study was supported by the Cystic Fibrosis Foundation (GORALS12LO and BUTTON07XX0) and the National Institutes of Health (1R01HL125280-01A1 and P30DK065988-11). Funding information for this article has been deposited with the Crossref Funder Registry.

\section{References}

1 Boucher RC. Evidence for airway surface dehydration as the initiating event in CF airway disease. J Intern Med 2007; 261: 5-16.

2 Button B, Cai LH, Ehre C, et al. A periciliary brush promotes the lung health by separating the mucus layer from airway epithelia. Science 2012; 337: 937-941.

3 Bush A, Payne D, Pike S, et al. Mucus properties in children with primary ciliary dyskinesia: comparison with cystic fibrosis. Chest 2006; 129: 118-123.

4 Redding GJ, Kishioka C, Martinez P, et al. Physical and transport properties of sputum from children with idiopathic bronchiectasis. Chest 2008; 134: 1129-1134.

5 Donaldson SH, Bennett WD, Zeman KL, et al. Mucus clearance and lung function in cystic fibrosis with hypertonic saline. N Engl J Med 2006; 354: 241-250.

6 Elkins MR, Robinson M, Rose BR, et al. A controlled trial of long-term inhaled hypertonic saline in patients with cystic fibrosis. N Engl J Med 2006; 354: 229-240.

7 Tarrant BJ, Le Maitre C, Romero L, et al. Mucoactive agents for chronic, non-cystic fibrosis lung disease: a systematic review and meta-analysis. Respirology 2017; 22: 1084-1092. 
8 Kellett F, Robert NM. Nebulised 7\% hypertonic saline improves lung function and quality of life in bronchiectasis Respir Med 2011; 105: 1831-1835.

9 Tang XX, Ostedgaard LS, Hoegger MJ, et al. Acidic pH increases airway surface liquid viscosity in cystic fibrosis. J Clin Invest 2016; 126: 879-891.

10 Tarran R, Grubb BR, Parsons D, et al. The CF salt controversy: in vivo observations and therapeutic approaches. Mol Cell 2001; 8: 149-158.

11 Goralski JL, Button B. Mucus concentration affects HBE response to nebulized hypertonic saline. Pediatr Pulmonol 2014; 49: 182.

12 Goralski JL, Button B. Nebulized hypertonic saline yields disparate ASL heights with sequential doses. Pediatr Pulmonol 2013; 48: 107.

13 Goralski JL, Button B. CF cells do not hyperabsorb sodium in the setting of nebulized hypertonic saline. Pediatr Pulmonol 2012; 34: 105.

14 Goralski JL, Button B. An in vitro study of the kinetics of hypertonic saline on ASL height. Pediatr Pulmonol 2010; 47: 205.

15 Thelin W, Donn K, Ansede J, et al. The ENaC inhibitor P-1037 is a CFTR-independent therapeutic agent that promotes sustained airways hydration and mucociliary transport. Pediatr Pulmonol 2015; 50: 201.

16 Warren NJ, Tawhai MH, Crampin EJ. A mathematical model of calcium-induced fluid secretion in airway epithelium. J Theor Biol 2009; 259: 837-849.

17 Wu D, Miyawaki S, Tawhai MH, et al. A numerical study of water loss rate distributions in MDCT-based human airway models. Ann Biomed Eng 2015; 43: 2708-2721.

18 Wu D, Boucher RC, Button B, et al. An integrated mathematical epithelial cell model for airway surface liquid regulation by mechanical forces. J Theor Biol 2018; 438: 34-45.

19 Blackmon RL, Kreda SM, Sears PR, et al. Direct monitoring of pulmonary disease treatment biomarkers using plasmonic gold nanorods with diffusion-sensitive OCT. Nanoscale 2017; 9: 4907-4917.

20 Fischbarg J. Fluid transport across leaky epithelia: central role of the tight junction and supporting role of aquaporins. Physiol Rev 2010; 90: 1271-1290.

21 Willumsen NJ, Davis CW, Boucher RC. Selective response of human airway epithelia to luminal but not serosal solution hypertonicity. Possible role for proximal airway epithelia as an osmolality transducer. J Clin Invest 1994; 94: 779-787.

22 Rasgado-Flores H, Krishna Mandava V, Siman H, et al. Effect of apical hyperosmotic sodium challenge and amiloride on sodium transport in human bronchial epithelial cells from cystic fibrosis donors. Am J Physiol Cell Physiol 2013; 305: C1114-C1122.

23 Herschlag G, Garcia GJ, Button B, et al. A mechanochemical model for auto-regulation of lung airway surface layer volume. J Theor Biol 2013; 325: 42-51.

24 Graeber SY, Zhou-Suckow Z, Schatterny J, et al. Hypertonic saline is effective in the prevention and treatment of mucus obstruction, but not airway inflammation, in mice with chronic obstructive lung disease. Am J Respir Cell Mol Biol 2013; 49: 410-417.

25 Tabary O, Escotte S, Couetil JP, et al. High susceptibility for cystic fibrosis human airway gland cells to produce IL-8 through the IKB kinase $\alpha$ pathway in response to extracellular $\mathrm{NaCl}$ content. J Immunol 2000; 164: $3377-3384$.

26 Hashimoto S, Matsumoto K, Gon Y, et al. Hyperosmolarity-induced interleukin-8 expression in human bronchia epithelial cells through p38 mitogen-activated protein kinase. Am J Respir Crit Care Med 1999; 159: 634-640.

27 Yukutake Y, Tsuji S, Hirano Y, et al. Mercury chloride decreases the water permeability of aquaporin-4-reconstituted proteoliposomes. Biol Cell 2008; 100: 355-363.

28 Kuwahara M, Gu Y, Ishibashi $\mathrm{K}$, et al. Mercury-sensitive residues and pore site in AQP3 water channel. Biochemistry 1997; 36: 13973-13978.

29 Vergilio CS, Carvalho CE, Melo EJ. Mercury-induced dysfunctions in multiple organelles leading to cell death. Toxicol In Vitro 2015; 29: 63-71.

30 Henderson AG, Ehre C, Button B, et al. Cystic fibrosis airway secretions exhibit mucin hyperconcentration and increased osmotic pressure. J Clin Invest 2014; 124: 3047-3060.

31 Morgan KS, Donnelley M, Farrow N, et al. In vivo X-ray imaging reveals improved airway surface hydration after a therapy designed for cystic fibrosis. Am J Respir Crit Care Med 2014; 190: 469-471.

32 Blackmon RL, Kreda SM, Sears PR, et al. Diffusion-sensitive optical coherence tomography for real-time monitoring of mucus thinning treatments. Proc SPIE Int Soc Opt Eng 2016; 9697: 969724.

33 Henderson AG, Bennett WD, Zeman KL, et al. Effects of inhaled hypertonic saline on mucociliary clearance and clinical outcomes in patients with chronic bronchitis. Am J Respir Crit Care Med 2017; 195: A6455.

34 Sandefur CI, Boucher RC, Elston TC. Mathematical model reveals role of nucleotide signaling in airway surface liquid homeostasis and its dysregulation in cystic fibrosis. Proc Natl Acad Sci USA 2017; 114: E7272-E7281.

35 Bennett WD, Wu J, Fuller F, et al. Duration of action of hypertonic saline on mucociliary clearance in the normal lung. J Appl Physiol 2015; 118: 1483-1490.

36 Locke LW, Myerburg MM, Markovetz MR, et al. Quantitative imaging of airway liquid absorption in cystic fibrosis. Eur Respir J 2014; 44: 675-684 